\title{
STANDARDIZED ELECTROMYOGRAPHIC INDEXES ALLOW A RELIABLE MEASUREMENT OF MASTICATORY MUSCLES FUNCTION
}

\author{
Riccardo Rosati ${ }^{1 a^{*}}$, Ekaterina Semelewa ${ }^{2 b}$, Elena Satygo ${ }^{2 c}$, Elena P. Ivanova ${ }^{3 \mathrm{~d}}$, Francesco Rosati ${ }^{4 \mathrm{e}}$, Alexey Silin ${ }^{2 c}$, \\ Virgilio F. Ferrario ${ }^{1 \mathrm{~b}}$ \\ 'Università degli Studi di Milano, Milano, Italy \\ ${ }^{2}$ North-Western State Medical University named after I.I. Mechnikov, Saint-Petersburg, Russian Federation \\ ${ }^{3}$ Moscow State University of Medicine and Dentistry named after A. I. Evdokimov, Moscow, Russian Federation \\ 4Private Practice, Udine, Italy \\ ${ }^{\mathrm{a} D D S}, \mathrm{PhD}$ \\ ${ }^{b} \mathrm{MD}, \mathrm{PhD}$ \\ 'MD, DMD \\ dDMD, PhD \\ ${ }^{\mathrm{e}} \mathrm{CDT}$, Private Practitioner
}

Received: March 15, 2016

Revised: June 04,2016

Accepted: July 11, 2016

Published: July 14, 2016

Cite this article:

Rosati R, Semelewa E, Satygo E, Ivanova EP, Rosati F, Silin A, Ferrario VF. Standardized electromyographic indexes allow a reliable measurement of masticatory muscles function. Stoma Edu J. 2016;3(3-4):235-242.

Introduction: Surface electromyography (sEMG) allows the quantitative and qualitative analysis of muscles recruitment and it is a method currently used in research and in the clinical practice. Considering that sEMG assesses the portion of muscular electrical activity that reaches cutaneous surface, a well-standardized protocol should be used, allowing to remove (or reduce) technical and biological artefacts.

Methodology: To evaluate the repeatability of the normalized indexes describing masticatory muscles sEMG, the standardized cutaneous myoelectric activity of Temporalis Anterior and Masseter muscles was recorded twice during a one week interval (Acquisition sessions T1 and T2) in 20 young healthy adults. The data obtained during T1 and T2 were compared. A clinical case was shown as an example of clinical sEMG application.

Results: No significant differences in the standardized indexes elaborated by sEMG signals of T1 and T2 acquisition sessions were found, strengthening the effectiveness of the standardization procedure.

Conclusion: sEMG indexes allow the evaluation of occlusal-induced proprioceptive mediated muscular recruitment in a reliable way. This measurement protocol can be clinically applied to estimate the muscular adaption to new occlusal conditions or to re-establish physiological muscular coordination.

Keywords: sEMG, dental proprioception, occlusion, oral rehabilitation, overlay.

\section{Introduction}

Every day the stomatognathic apparatus constantly performs many physiologic activities, such as speech, mastication, suction, deglutition. The muscles responsible for the mandibular, tongue, hyoid, soft palate, lips movements generate mechanical forces that are dissipated by the hard tissues (bones, temporomandibular joint, teeth). In all these functions several muscle groups are involved and coordinated by the central nervous system under the influence of peripheral inputs. Surface electromyography (sEMG) is a low-cost, noninvasive method usable in research and in dental clinical practice for the quantitative and qualitative analysis of head and neck muscles ${ }^{1-23}$. As sEMG does not directly assess the muscular fibers, a wellstandardized protocol should be used, allowing to remove (or reduce) technical and biological artefacts. Indeed, the thickness of the hypodermis, crosstalk from different muscles, the instrumental noise and the position of the electrodes relative to the muscle fibers and the motor point can influence the sEMG signal. Taking into account these technical features, a correct sEMG assessment should be performed only with a reproducible protocol, and with standardized normalized potentials to remove most of biological and technical noise $2,3,8,9,10,13$. The aim of the present study was to evaluate the repeatability of masticatory muscles normalized indexes obtained during maximal voluntary clenching sEMG acquisitions.

\section{*Corresponding author:}

Riccardo Rosati, DDS, PhD, Università degli Studi di Milano, Via Luigi Mangiagalli 31, I-20133 Milano, Italy Phone: +39 02 50315390, Fax: +39 02 50315387, e-mail: riccardo@riccardorosati.eu 


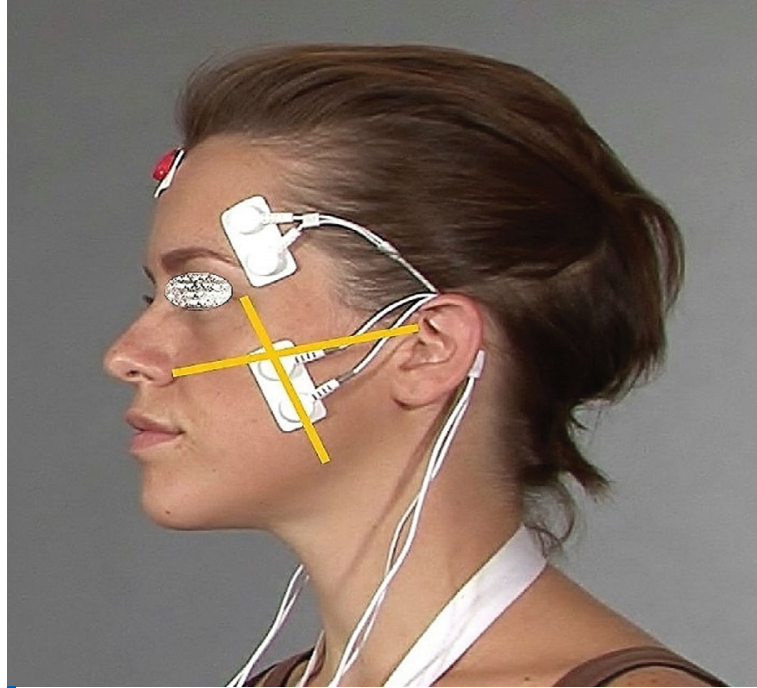

Figure 1. Masseter and Temporalis Anterior electrode placement

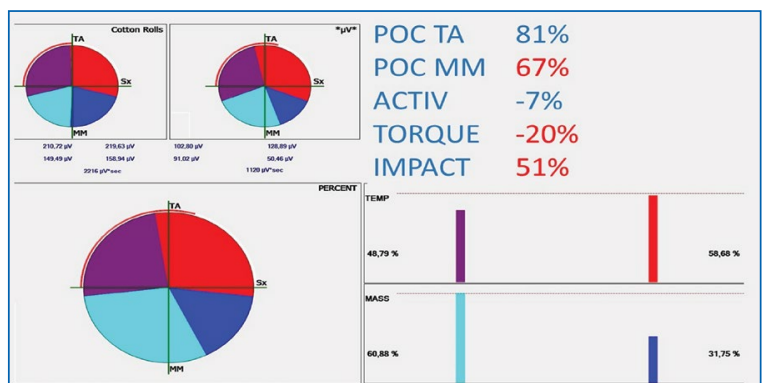

Figure 3. Initial sEMG differential test. Reported indexes are: Temporalis Anterior and Masseter percentage overlapping coefficient (POC TA and POC MM); activity index (ACTIV); torque coefficient (TORQUE) and standardized activity index (IMPACT)

\section{Methodology}

\subsection{Study design}

Standardized cutaneous myoelectric activity of Temporalis Anterior and Masseter muscles was acquired twice with a one-week interval (Acquisition sessions T1 and T2) in 20 young healthy adults. The data obtained during T1 and T2 were compared.

\subsection{Subjects}

Twenty young healthy adult volunteers ( 5 males, 15 females, age range 24-28 years, mean 26, SD $2)$, volunteers were recruited for the study after a detailed explanation of the experimental protocol and possible risks involved.

They all gave their consent to the investigation according to the principles outlined in the Declaration of Helsinki. The study protocol was approved by the local ethic committee. All data collections were performed in the clinic of Dental Faculty of North-Western State Medical University named after I.I. Mechnikov (Saint Petersburg, Russian Federation).

The subjects were visited by a dentist (SE); all the subjects were in general good health, free from stomatognathic apparatus and neck pathologies

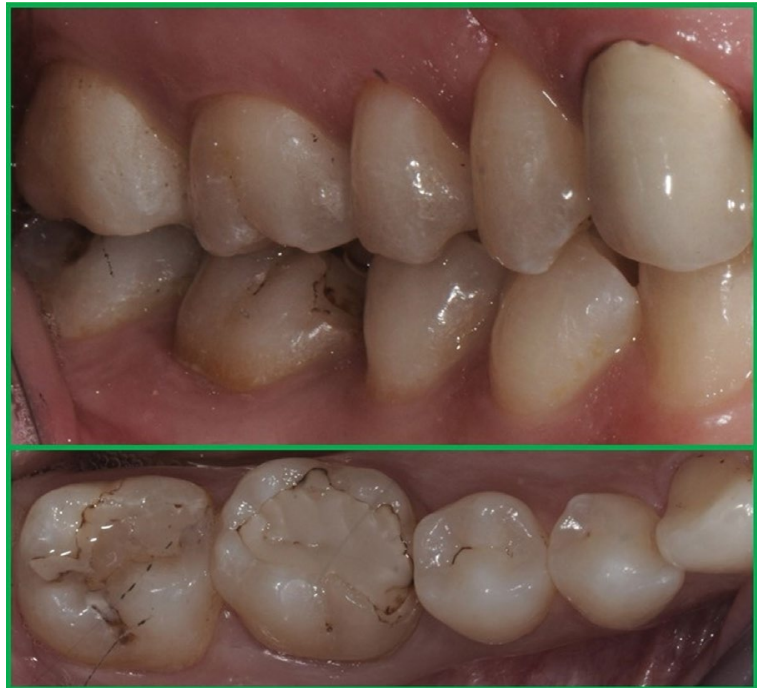

Figure 2. Initial clinical condition

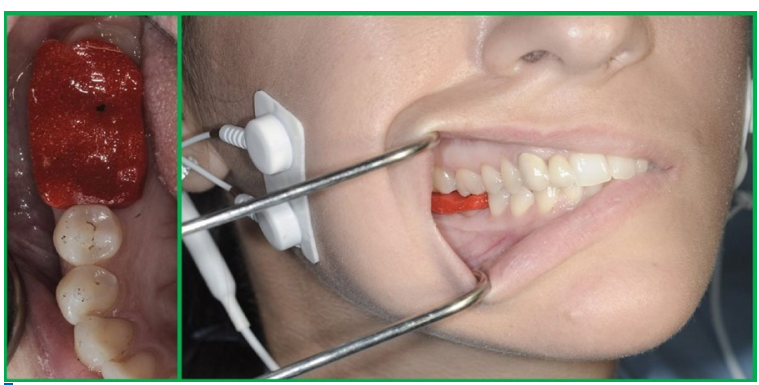

Figure 4. Resin jig used to arrange vertical dimension following muscle performance

and were selected for the study according to the following inclusion criteria: a minimum of 28 permanent teeth, no parodontal problems, no craniofacial and cervical trauma and surgery, no temporo-mandibular and craniocervical disorders, no current orthodontic treatment and no painful sensation when clenching their teeth. The subjects were excluded from the study if they had neurological problems that could interfere with the experimental procedure, or if they were taking drugs that could affect the musculoskeletal system, such as anti-inflammatory or pain relief drugs. During the sEMG recording, the environment was quiet and with low light. The subjects sat in a comfortable office type chair, in an erect posture with their feet flat on the floor, and arms resting on their legs.

\subsection{Electrode type and positioning}

The Masseter (MM) and Temporalis Anterior (TA) muscles of both sides (left and right) were examined. Disposable pre-gelled silver/silver chloride bipolar surface electrodes (rectangular shape, $21 \times 41 \mathrm{~mm}, 20 \mathrm{~mm}$ inter-electrode distance) (F3010, Fiab, Firenze, Italy) were positioned. The 
Table 1. Comparison of standardized sEMG indexes obtained in two different data collection sessions (all values are percentages). No statistically significant differences were found

\begin{tabular}{|c|c|c|c|c|c|}
\hline \multicolumn{6}{|c|}{ Masticatory sEMG standardized indexes repeatability } \\
\hline & POCTA & POC MM & Activity & Torque & Impact \\
\hline \multicolumn{6}{|l|}{ Session 1} \\
\hline Mean & 82.3 & 84.5 & 1.5 & -1.0 & 114.2 \\
\hline SD & 1.3 & 1.2 & 4.6 & 4.0 & 16.3 \\
\hline \multicolumn{6}{|l|}{ Session 2} \\
\hline Mean & 82.0 & 84.4 & 1.1 & 0.6 & 113.5 \\
\hline SD & 0.8 & 1.1 & 4.7 & 3.2 & 12.5 \\
\hline \multicolumn{6}{|l|}{ Difference } \\
\hline Mean & 0.2 & 0.1 & 0.4 & -1.5 & 0.7 \\
\hline SD & 0.8 & 0.7 & 4.1 & 4.8 & 13.7 \\
\hline TTest & 0.166 & 0.408 & 0.691 & 0.166 & 0.821 \\
\hline
\end{tabular}

electrodes were placed on the muscular bellies parallel to muscular fibres as follows (Fig. 1):

- MM: the operator, standing in front of the seated subject, palpated the muscular belly while the subject clenched his/her teeth. The electrodes were fixed parallel to the exocanthion-gonion line and with the upper pole of the electrode under the tragus-labial commissura line.

- TA: the muscular belly was palpated during tooth clenching and the electrodes were fixed vertically along the anterior margin of the muscle (corresponding to the fronto-parietal suture $)^{24}$.

A disposable reference electrode was applied to the forehead. To reduce skin impedance, the skin was carefully cleaned prior to the electrode placement, and recordings were performed 5 min later, allowing the conductive paste to adequately moisten the skin.

\section{4. sEMG recordings and measurements Instrumentation}

The surface EMG activity was recorded using a computerized instrument (Easymyo, 3 Technology S.r.l., Udine, Italy). The analogic sEMG signal was amplified (gain 100, bandwidth 0-1000 Hz, peakto-peak input range from 0 to $3600 \mu \mathrm{V}$ ) using a differential amplifier with a high common mode rejection ratio $(C M R R=115 \mathrm{~dB}$ in the range $0-60$ $\mathrm{Hz}$, input impedance $100 \mathrm{G} \Omega$ ), digitized (24-bit resolution, $4000 \mathrm{~Hz}$ A/D sampling frequency), and digitally filtered (Butterworth type, high-pass filter set at $30 \mathrm{~Hz}$, low-pass filter set at $400 \mathrm{~Hz}$, band-stop for common $50-60 \mathrm{~Hz}$ noise). The signals were averaged over $25 \mathrm{~ms}$, with muscle activity assessed as the root mean square (RMS) of the amplitude ( $\mu \mathrm{V})$. sEMG signals were recorded for further analysis. Before the acquisition session the subjects were properly trained to elicit true teeth maximal voluntary contraction using an on-time sEMG signal visualization.

\section{Results}

\subsection{Standardization procedure}

To normalize the sEMG signals two $10-\mathrm{mm}$ thick cotton rolls were positioned on the mandibular second premolars/first molars of each subject and a 5-s maximum voluntary contraction (MVC) was recorded. The mean sEMG potential of each muscle obtained in that first acquisition was set at $100 \%$, and all further sEMG potentials were expressed as a percentage of this value $(\mu \mathrm{V} /$ $\mu \mathrm{V} \times 100)$

\subsection{Analyzed task}

The sEMG activity was recorded during a 5-s MVC test in intercuspal position (IP): the subject was invited to clench as hard as possible and to maintain the same level of contraction for all the test. For each patient, the central $3 \mathrm{~s}$ of the MVC test were analysed, and the sEMG potential was standardized as detailed before.

\section{3. sEMG data analysis}

Separately for each acquisition session (T1 and T2), the sEMG waves were compared by computing a series of standardized indexes using the instrument software tools:

1. the percentage overlapping coefficient (POC, unit $\%)$, an index of symmetric muscular contraction. The index ranges between $0 \%$ and 100\%: when two paired muscles contract with perfect symmetry, a POC of $100 \%$ is obtained. Masseter and Temporalis Anterior POCs were obtained for each patient ${ }^{24,25}$.

2. the torque coefficient (TORQUE, unit \%) was assessed to evaluate if an unbalanced contractile activity of the contralateral Masseter and Temporalis Anterior muscles, such as that of the right Temporalis Anterior and the left Masseter, might give rise to a potential lateral displacing component. TORQUE ranges between 100\% (complete prevalence of the right Temporalis Anterior and left Masseter) and $-100 \%$ (complete prevalence of the left Temporalis Anterior and the right Masseter $)^{24,25}$.

3. the activity index (ATTIV, unit \%), was obtained as the percentage ratio of the difference between the mean Masseter and Temporalis 


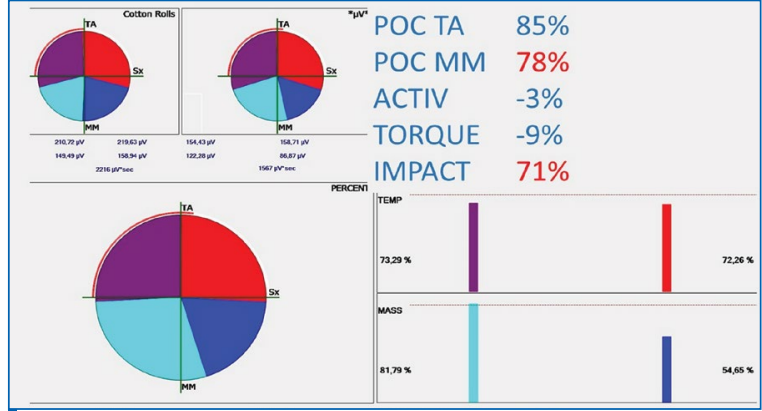

Figure 5. First test clenching wearing the jig

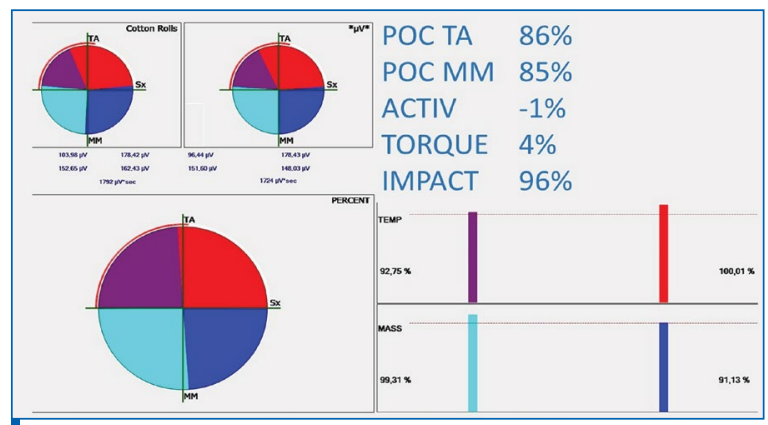

Figure 7. Final sEMG test clenching with cemented overlay

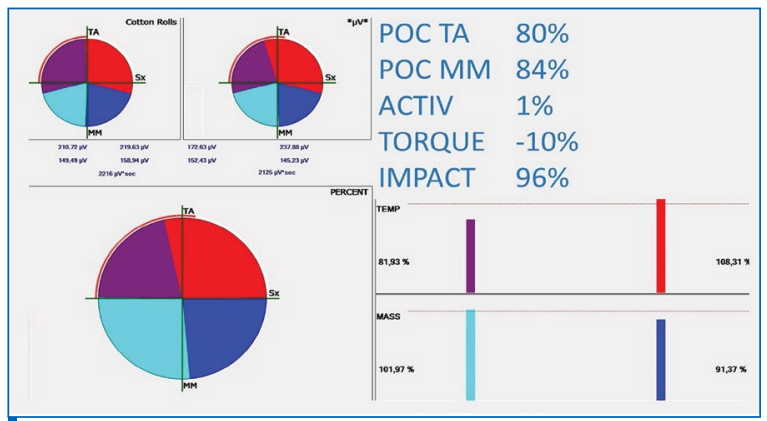

Figure 6. Final test wearing the (modified) jig

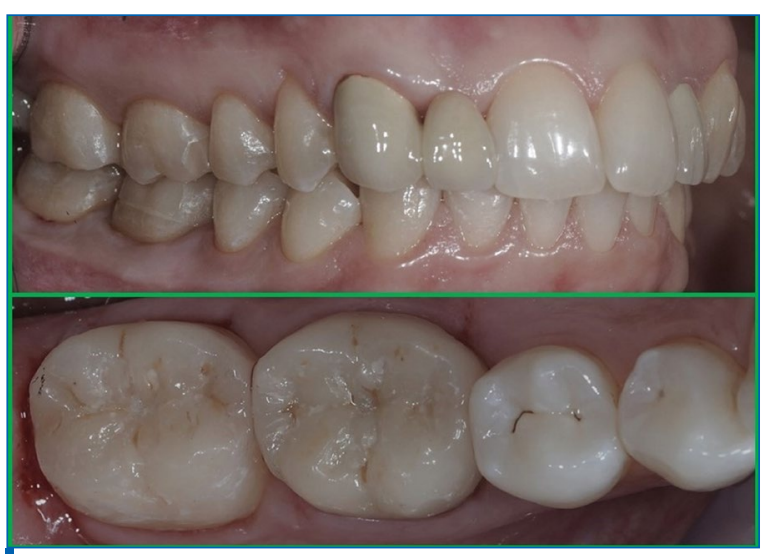

Figure 8. Final reconstructions
Anterior muscles standardized potentials and the sum of the same standardized potentials, to individuate the most prevalent pair of masticatory muscles. The index is positive (up to $100 \%$ ) if the Masseter muscles standardized potentials are larger than those of the Temporalis Anterior muscles, negative (up to $100 \%$ ) if the Temporalis Anterior muscle potentials are larger ${ }^{26}$.

4. the standardized activity index (IMPACT, unit \%*s) was calculated to quantify the total muscular activity performed during MVC relative to the standardization clenching on cotton rolls; IMPACT was estimated computing the mean (Masseter and Temporalis Anterior) total muscle activities as the integrated areas of the sEMG potentials over time ${ }^{26}$.

\subsection{Statistical evaluation}

To quantify the acquisition session effect on the sEMG standardized indexes, the values obtained from T1 and T2 were compared. Normality data distribution was assessed using Shapiro-Wilk test. Paired Student's T test $(p<0.05)$ was used to evaluate the systematic error between the two muscular measurements.

Table 1 shows the mean values and standard deviation of the standardized indexes obtained in the two data acquisition sessions, together with the mean difference in individual values. Paired Student's $T$ tests found no statistically significant differences.

\section{Discussion}

The surface electromyogram comprises the sum of the electrical contributions produced by the active motor units as detected by cutaneous electrodes. Several parameters could be analysed in the captured electrical signal. The discharge rates, signal amplitude, spectral analysis could be investigated to estimate the neural control strategies involved in the muscles management. ${ }^{27}$ These instrumental assessments should be performed applying the correct protocols; in fact, the myoelectric activity signal may vary due to many factors such as change in the electrode location, change in the tissue properties, tissue temperature, muscle resting length, velocity of contraction and fiber type $\mathrm{e}^{27-30}$.

In the last 30 years the effect of electrodes position on conduction velocity estimation (CV), amplitude and spectral variables of the surface EMG has been addressed in a number of methodological and clinical publications taking into account muscles (or groups of muscles) ranging from the masticatory muscles to the muscles of the shoulder, of the arm and leg too ${ }^{31}$. Changing the electrode location through innervation zones to tendons affect the detected activity: signal spectrum shifts towards higher frequencies, the amplitude decreases and the conduction velocity is biased towards higher values ${ }^{27}$. Moreover we have to underline that surface electrodes, in many cases, 
capture electrical signals generated from several muscles -crosstalk effect- preventing a single muscle measurement. In facial cutaneous areas, the mimic muscles and sometimes anatomical variability, could complicate the univocal sEMG signal-muscle matching (as previously reported platysma to masseter partial coverage) $)^{32}$.

Indeed it is well known that some muscles functional compartmentalization due to their anatomical and innervation complexity thwarts a simple function assessment; in particular Temporalis muscles is a composite morphologic-functional entity not yet fully understood 33,34 .

In any case, taking into account the technical complications, it appears reasonable to apply sEMG analysis to understand the functional areas central management instead of focusing on single muscle function measurement.

Notwithstanding the amount of experimental and theoretical studies, no global consensus was achieved in the sEMG raw signal interpretation. For this reason, studies that could increase the understanding of sEMG advantages and limits in order to allow a more aware clinical application are required.

The most revealing standardization effort occurred in 1997-1999 within the European Project on "Surface EMG for Non Invasive Assessment of Muscles" where a fine analysis was presented for a number of sEMG acquisition technical factors $^{35,36}$. Yet, when comparing several EMG studies published in the year 2004, Armijo-Olivo et al. stated that $60 \%$ of the analysed studies did not report a normalization procedure, making comparisons of the EMG signal difficult due to anthropomorphic differences between recording sites and individuals ${ }^{37}$. They underlined that comparison of muscle function evaluating the absolute values of the recorded potentials (that is, microvolt values) provides inaccurate results. As a consequence of this inaccuracy, comparing the EMG activities between subjects and under different conditions requires a normalization process.

The efficacy of intra-subjects standardization procedure was investigated also for the jaw stretch reflex. In fact, Koutris et al. demonstrated that when the amplitude of the masseter muscle during the jaw stretch reflex is normalized relative to the prestimulus EMG amplitude, it becomes independent from the location of the electrodes over the muscle ${ }^{38}$. In the present study, the repeatability of standardized (normalized) indexes calculated in independent sEMG acquisitions was evaluated. Indeed, as suggested by Ferrario et al., sEMG could be usefully employed for the control of the muscular modifications induced by variations in the occlusal conditions, either natural (for instance, different occlusal classes, crossbite occlusion), or artificial (for instance, dental prostheses, fillings, or even orthodontic treatment ${ }^{26,39}$. The use of sEMG can help in the correct construction of oral devices ${ }^{40}$. To simplify the understanding of occlusal induced muscular modifications, easy-tointerpret indexes could be helpful in the clinical device/ prosthesis-adapting procedures. In order to perform a correct evaluation of these indexes in the everyday clinical practice, their reliability should be accurately understood.

No significant differences in the standardized indexes elaborated from sEMG signals of T1 and T2 acquisition sessions were found, strengthening the effectiveness of the standardization procedure. The small sample analysed in the present study does not allow the evaluation of the values in an epidemiologic perspective. The average POC of Temporalis Anterior and Masseter, obtained during this study, resulted slightly lower than the ones reported by De Felicio et al. and by Ferrario et al., probably due to differences in the selection of the sample 9,16,24.

As regards the standardization of masticatory muscles sEMG signal, a unique agreement within the scientific community does not exist ${ }^{28}$. Different sEMG studies have made use of the protocol and related indexes proposed by Ferrario et al. to evaluate the masticatory muscles ${ }^{16,24,41-44}$. The only difference between two acquisitions (on cotton rolls and in IP) is dental contact, thus limiting the technical and biological noise inherent in this instrumental analysis. This way, through the estimate of standardized indexes, it is possible to evaluate the muscular reaction to dental proprioception. Other stimuli, that can influence the muscular activity in both tests (for instance, intramuscular pain), cannot always be identified by evaluating the standardized indexes (supposing that they can influence the standardization task and the teeth clenching in the same way). For this reason, an effective dental occlusion instrumental evaluation applying sEMG protocol needs healthy supporting tissues (bones, teeth, periodontium). Applying these clinical recommendations, the proposed standardization approach allows dental proprioception effect on functional areas management isolation, reducing the crosstalk and technical signal confounding factors relevance.

Anyway, instrumental information about muscular functional adaptation at dental contact can be useful for the classification of dental patients, and the functionalization of therapeutic procedures as removable and fixed prostheses. As an example, patient L.D., a woman aged 26, needed rehabilitation of incongruous composite fillings (Fig. 2). An initial sEMG test could aid the Dentist to estimate if the reduced posterior vertical dimension, due to filling wear, can cause altered muscular performance. As shown in Fig. 3, the initial sEMG test revealed that the pre-operative occlusal condition did not allow a maximal muscular activity.

Masseter POC was lower than $80 \%$ as the result of a no-synchronous and asymmetric Masseter recruitment. This performance alteration hesitated in a torque effect during maximal voluntary clenching (Torque index -20\%). The Impact index 
confirmed that the occlusal condition needed muscular adaptation (while clenching in maximal intercuspation, the muscles performed only $51 \%$ of electrical activity developed while clenching with cotton rolls).

After a detailed explanation of the clinical and instrumental conditions, the patient agreed to rehabilitate her first and second right mandibular molars with overlays, changing her right posterior occlusal support.

During the final impression taking appointment, a stiff flat resin jig was set on reduced tooth substance to slightly increase the posterior vertical dimension. A sEMG acquisition was made, and the patient was invited to perform two maximal voluntary clench: the first one with cotton rolls between the dental arches and the second one wearing the jig (Fig. 4). Fig. 5 shows that the torque effect was reduced; muscular total work and Masseter coordination increased but did not reach physiologic values (Impact index from 51 to $71 \%$ and POC Masseter $78 \%$ ). The jig was then slightly reduced and retested obtaining a muscular recruitment very similar to those recorded in healthy young subjects with sound dentition (Fig. 6) 24,41-42. The new EMG tested mandibular position (wearing the modified jig) was used as occlusal posterior vertical index to produce the final indirect overlays.

During the subsequent appointment, the overlays were bonded in a dry setting and the function verified again acquiring muscle performance with the same standardized protocol.

The new posterior occlusal morphology well integrated in the patient stomatognathic structures, and she had a good masticatory muscles coordination: her performance with the new dental surfaces well overlapped the "occlusion-free" reference test (clench on cotton rolls) (Fig. 7). The functional test was satisfactory so reconstructions were accurately polished (Fig. 8).

\section{Conclusions}

Standardized sEMG indexes allow the evaluation of occlusal-induced proprioceptive mediated muscular recruitment in a reliable way.

Merging biomechanical concepts with sEMG standardized indexes, occlusal devices and prosthesis adapting procedures could be clinically performed in order to reduce the muscular adaption to the new occlusal conditions or to reestablish physiological muscular coordination ${ }^{45}$.

\section{Acknowledgments}

The authors declares no conflict of interest related to this study. There are no conflicts of interest and no financial interests to be disclosed.

\section{REFERENCES}

1. Dong Y, Wang XM, Wang MQ, Widmalm SE. Asymmetric muscle function in patients with developmental mandibular asymmetry. J Oral Rehabil. 2008;35(1):27-36.

2. Ferrario VF, Tartaglia GM, Luraghi FE, Sforza C. The use of surface electromyography as a tool in differentiating temporomandibular disorders from neck disorders. Man Ther. 2007;12(4):372-379.

3. Armijo-Olivo S, Magee DJ. Electromyographic activity of the masticatory and cervical muscles during resisted jaw opening movement. J Oral Rehabil. 2007;34(3):184-194.

4. Ceneviz C, Mehta NR, Forgione A, Sands MJ, Abdallah EF, Lobo Lobo S, Mavroudi S. The immediate effect of changing mandibular position on the EMG activity of the masseter, temporalis, sternocleidomastoid, and trapezius muscles. Cranio. 2006;24(4):237-244.

5. Santander H, Miralles R, Jimenez A, Zuniga C, Rocabado M, Moya $\mathrm{H}$. Influence of stabilization occlusal splint on cranio-cervical relationships. Part II: Electromyographic analysis. Cranio. 1994;12(4):227-233.

6. Zuniga C, Miralles R, Mena B, Mont R, Moran D, Santander $H$, Moya $H$. Influence of variation in jaw posture on sternocleidomastoid and trapezius electromyographic activity. Cranio. 1995;13(3):157-162.

7. Ciuffolo F, Manzoli L, Ferritto AL, Tecco S, D'Attilio M, Festa F. Surface electromyographic response of the neck muscles to maximal voluntary clenching of the teeth. J Oral Rehabil. 2005;32(2):7984.

8. Ferrario VF, Sforza C, Dellavia C, Tartaglia GM. Evidence of an influence of asymmetrical occlusal interferences on the activity of the sternocleidomastoid muscle. J Oral Rehabil. 2003;30(1):34-40.

9. Ferrario VF, Tartaglia GM, Galletta A, Grassi GP, Sforza C. The influence of occlusion on jaw and neck muscle activity: a surface EMG study in healthy young adults. J Oral Rehabil. 2006;33(5):341-348.

10. Sforza C, Tartaglia GM, Solimene U, Morgun V, Kaspranskiy RR, Ferrario VF. Occlusion, sternocleidomastoid muscle activity, and body sway: a pilot study in male astronauts. Cranio. 2006;24(1):43-49.

11. Tartaglia GM, Testori T, Pallavera A, Marelli B, Sforza C. Electromyographic analysis of masticatory and neck muscles in subjects with natural dentition, teeth-supported and implant-supported prostheses. Clin Oral Implants Res. 2008;19(10):1081-1088.

12. Tecco S, Caputi S, Festa F. Electromyographic activity of masticatory, neck and trunk muscles of subjects with different skeletal facial morphology -- a cross-sectional evaluation. J Oral Rehabil. 2007;34(7):478-486.

13. Forrester SE, Allen SJ, Presswood RG, Toy AC, Pain MT. Neuromuscular function in healthy occlusion. J Oral Rehabil. 2010;37(9):663-669. 
14. Castroflorio T, Farina D, Bottin A, Piancino MG, Bracco $P$, Merletti R. Surface EMG of jaw elevator muscles: effect of electrode location and inter-electrode distance. J Oral Rehabil. 2005;32(6):411-417.

15. Castroflorio T, Bracco P, Farina D. Surface electromyography in the assessment of jaw elevator muscles. J Oral Rehabil. 2008;35(8):638-645

16. De Felıcio CM, Sidequersky FV, Tartaglia GM, Sforza C.

Electromyographic standardized indices in healthy Brazilian young adults and data reproducibility. J Oral Rehabil. 2009;36(8):577-583. 17. Ekstrom RA, Soderberg GL, Donatelli RA. Normalization procedures using maximum voluntary isometric contractions for the serratus anterior and trapezius muscles during surface EMG analysis. J Electromyogr Kinesiol. 2005;15(4):418-428.

18. Farina D, Madeleine P, Graven-Nielsen T, Merletti R, ArendtNielsen L. Standardising surface electromyogram recordings for assessment of activity and fatigue in the human upper trapezius muscle. Eur J Appl Physiol. 2002;86(6):469-478.

19. McLean L, Chislett M, Keith M, Murphy M, Walton P. The effect of head position, electrode site, movement and smoothing window in the determination of a reliable maximum voluntary activation of the upper trapezius muscle. J Electromyogr Kinesiol. 2003;13(2):169-180.

20. Suvinen TI, Malmberg J, Forster C, Kemppainen P. Postural and dynamic masseter and anterior temporalis muscle EMG repeatability in serial assessments. J Oral Rehabil. 2009;36(11):814-820.

21. Tartaglia GM, Moreira Rodrigues da Silva MA, Bottini $S$, Sforza C, Ferrario VF. Masticatory muscle activity during maximum voluntary clench in different research diagnostic criteria for temporomandibular disorders (RDCTMD) groups. Man Ther. 2008;13(5):434-440.

22. Learreta JA, Beas J, Bono AE, Durst A. Muscular activity disorders in relation to intentional occlusal interferences. Cranio. 2007;25(3):193-199.

23. Cecílio FA, Regalo SC, Palinkas M, Issa JP, Siéssere $S$, Hallak JE, Machado-de-Sousa JP, Semprini M. Ageing and surface EMG activity patterns of masticatory muscles. J Oral Rehabil. 2010;37(4):248-255.

24. Ferrario VF, Sforza C, Colombo A, Ciusa V. An electromyographic investigation of masticatory muscles symmetry in normo-occlusion subjects. J Oral Rehabil. 2000;27(1):33-40.

25. Ferrario VF, Sforza C, Serrao G, Colombo A, Schmitz JH. The effects of a single intercuspal interference on electromyographic characteristics of human masticatory muscles during maximal voluntary teeth clenching. Cranio. 1999;17(3):184-188.

26. Ferrario VF, Sforza C, Tartaglia GM, Dellavia C. Immediate effect of a stabilization splint on masticatory muscle activity in temporomandibular disorder patients. J Oral Rehabil. 2002;29(9):810-815. 27. Farina D, Cescon C, Merletti R. Influence of anatomical, physical, and detection-system parameters on surface EMG. Biol Cybem. 2002;86(6):445-456.

28. Burden A, Bartlett R. Normalisation of EMG amplitude: an evaluation and comparison of old and new methods. Med Eng Phys. 1999;21(4):247-257.
29. Lapatki BG, Oostenveld R, Van Dijk JP, Jonas IE, Zwarts MJ, Stegeman DF. Optimal placement of bipolar surface EMG electrodes in the face based on single motor unit analysis. Psychophysiology. 2010;47(2):299-314.

30. Campanini I, Merlo A, Degola P, Merletti R, Vezzosi G, Farina D. Effect of electrode location on EMG signal envelope in leg muscles during gait. J Electromyogr Kinesiol. 2007;17(4):515-526.

31. Mesin L, Merletti R, Rainoldi A. Surface EMG: the issue of electrode location. J Electromyogr Kinesiol. 2009;19(5):719-726.

32. Widmalm SE, Nemeth PA, Ash MM Jr, Lillie JH. The anatomy and electrical activity of the platysma muscle. J Oral Rehabil. 1985;12(1):17-22.

33. Chang Y, Cantelmi D, Wisco JJ, Fattah A, Hannam AG, Agur AM. Evidence for the functional compartmentalization of the temporalis muscle: a 3-dimensional study of innervation. J Oral Maxillofac Surg. 2013;71(7):1170-1177.

34. Benninger B, Lee BI. Clinical importance of morphology and nomenclature of distal attachment of temporalis tendon. J Oral Maxillofac Surg. 2012;70(3):557-561.

35. www.seniam.org. 2016 Mar 19

36. Hermens HJ, Freriks B, Disselhorst-Klug C, Rau G. Development of recommendations for SEMG sensors and sensor placement procedures. J Electromyogr Kinesiol. 2000;10(5):361-374. 37. Armijo-Olivo S, Gadotti I, Kornerup M, Lagravère MO, FloresMir C. Quality of reporting masticatory muscle electromyography in 2004: a systematic review. J Oral Rehabil. 2007;34(6):397-405. 38. Koutris M, Naeije M, Lobbezoo F, Wang K, Arendt-Nielsen L, Svensson P, Farina D. Normalization reduces the spatial dependency of the jaw-stretch reflex activity in the human masseter muscle. Muscle Nerve. 2010;41(1):78-84.

39. Wang MQ, He JJ, Zhang JH, Wang K, Svensson P, Widmalm SE. sEMG activity of jaw-closing muscles during biting with different unilateral occlusal supports. J Oral Rehabil. 2010;37(9):719-725. 40. Tartaglia GM, Sforza C. Functional evaluation of implant supported prostheses. Stoma Edu J. 2014;1(1):41-47.

41. Botelho AL, Gentil FH, Sforza C, da Silva MA. Standardization of the electromyographic signal through the maximum isometric voluntary contraction. Cranio. 2011;29(1):23-31.

42. Botelho AL, Melchior Mde O, da Silva AM, da Silva MA. Electromyographic evaluation of neuromuscular coordination of subject after orthodontic intervention. Cranio. 2009;27(3):152 158.

43. Di Palma E, Gasparini G, Pelo S, Tartaglia GM, Chimenti C. Activities of masticatory muscles in patients after orthognathic surgery. J Craniomaxillofac Surg. 2009;37(7):417-420. 44. Peretta R, Melison M, Meneghello R, Comelli D, Guarda L, Galzignato PF, Concheri G, Ferronato G. Unilateral masseter muscle hypertrophy: morphofunctional analysis of the relapse after treatment with botulinum toxin. Cranio. 2009;27(3):200210.

45. Ferrario VF, Sforza C. Biomechanical model of the human mandible in unilateral clench: distribution of temporomandibular joint reaction forces between working and balancing sides. J Prosthet Dent. 1994;72(2):169-176. 
Riccardo ROSATI

DDS, PhD

Functional Anatomy Research Center (FARC) Università degli Studi di Milano, Milano, Italy

Riccardo Rosati, CDT, received his DDS degree and PhD at the Università degli Studi di Milano, Italy. In 2011 he obtained a postgraduate degree in "Endodontology and Restaurative Dentistry" at Università degli Studi di Siena. He is involved in facial morphology and masticatory muscles function research. All investigations were developed using non-invasive instruments allowing healthy subjects measurements to obtain morphofunctional reference values. He is currently is a staff member researcher of the Functional Anatomy Research Center (FARC) of the Università degli Studi di Milano. He works as dentist in Milano and Udine focused on prosthetic reconstructions.

\section{0restions}

\section{Which factors could influence the surface electromyographic signal?}

$\square$ a. the thickness of the hypodermis;

$\square$ b. the instrumental noise;

ac. the position of the electrodes relative to the muscle fibres;

$\square$ d. all the previous factors.

\section{The Percentage Overlapping Coefficient:}

a. it measures the muscle force;

ab. it quantifies the symmetry of muscles contraction;

ac. it could be greater than $100 \%$;

$\square d$. it is always lower than $50 \%$.

\section{The Surface electromyography:}

$\square$ a. it uses needle electrodes inserted into muscle belly;

ab. it is performed applying electrodes on cutaneous surface;

ac. it is painful;

$\square$ d. it is invasive.

\section{The Raw electromyographic signal, standardized on clenching with cotton rolls, allows:}

$\square$ a. the muscle pain measurement;

$\square$ b. the dental proprioception related muscle reaction measurement;

$\square$ c. the muscle force evaluation;

$\square \mathrm{d}$. the TMJ position analysis. 